\title{
Special issue on canine genetics: animal models for human disease and gene therapies, new discoveries for canine inherited diseases, and standards and guidelines for clinical genetic testing for domestic dogs
}

\author{
Lisa G. Shaffer ${ }^{1}$ (]) \\ Received: 24 April 2019 / Accepted: 30 April 2019 / Published online: 5 May 2019 \\ (c) Springer-Verlag GmbH Germany, part of Springer Nature 2019
}

Keywords Dog $\cdot$ Canine $\cdot$ Genetic testing $\cdot$ Canine genetics $\cdot$ Dog genetics $\cdot$ Genetic mutation $\cdot$ Gene therapy $\cdot$ Genetic variants

\section{Introduction}

Dog domestication over several thousands of years and artificial selection for desired traits during breed development have inadvertently retained deleterious disease-associated mutations in certain dog populations. Although some disease variants occur in a single breed, many occur in several breeds either indicating a single ancestral founder with the mutation or crossbreeding between breeds with the mutation prior to the designation of the founder stock. Once a foundation stock has been designated, crossbreeding between breeds usually ceases to establish the desired physical and behavioral characteristics for that specific breed. The presence and frequencies of specific mutations and their association with disease vary among the different dog breeds depending on when the mutation occurred during breed development. Depending on the breed and disease, frequencies of carriers range from common to exceedingly, but measurably, rare.

In addition to being an important animal model for human genetic disease, the domestic dog has many jobs within our society by providing assistance, security and companionship. In recent years, especially since the sequencing of the canine genome (Lindblad-Toh et al. 2005), more than 350 likely causal variants have been identified to be associated with specific diseases and traits in the domestic dog (Online Mendelian Inheritance in Animals 2019). This special issue

Lisa G. Shaffer

Lshaffer@pawprintgenetics.com

1 Paw Print Genetics, Genetic Veterinary Sciences, Inc., 220 E Rowan, Suite 220, Spokane, WA 99207, USA of Human Genetics, dedicated to canine genetics, provides many excellent examples of the exciting discoveries taking place in the field of canine genomics.

\section{Animal models for human disease and gene therapies}

Progressive retinal atrophy (PRA) in dogs, referred to as retinitis pigmentosa in humans, represents high heterogeneity, as it is not a single disease but characterizes the clinical findings for which the genetic basis has been identified for more than 90 genes in dogs. Bunel et al. (2019) in their review of the genetic basis of PRA in dogs describe the work to date to identify the genetic causes of this common inherited condition and nicely illustrate how the dog has served and continues to serve as an important animal model for humans. In addition, their work in the Border Collie for an X-linked PRA demonstrates that even though certain dog breeds are highly inbred, some mutations can remain elusive and difficult to identify in this model organism.

Hereditary sensory neuropathies (HSN), also known as hereditary sensory and autonomic neuropathies in humans, constitute a clinically and genetically heterogeneous group of disorders. In dogs, about a dozen different breeds have been identified that develop sensory neuropathies. In the review by Corréard et al. (2019), various forms of human and canine HSN conditions are examined and compared. As well described by the authors, several conditions in dogs can serve as models of human disease and may lead to the identification of the causative genomic variants in both species. The availability of natural animal models, such as dogs, 
represents opportunities to unravel genetics and therapies for humans and dogs (Corréard et al. 2019).

Variation in drug response, whether manifesting as toxicity or failure to respond, occurs in dogs as it does in humans. Although the field of pharmacogenomics in companion animals lags behind what is understood in humans, Mealey et al. (2019), in this issue of Human Genetics, present a comprehensive review of what is known today for the domestic dog. Advances made in the field of canine pharmacogenomics may not only improve the health and welfare of dogs but may provide helpful insights into human variation in drug effectiveness, response and potential for adverse reactions.

Therapeutic approaches for inherited diseases include dietary changes or restrictions, proteins or products synthesized through recombinant DNA technologies, enzyme replacement, and various gene therapy and gene editing strategies. Using an animal model can help inform approaches for human therapies for a similar or identical disorder. Duchenne muscular dystrophy (DMD) is an example of an inherited disease found in humans that occurs as a spontaneous animal model in dogs. As reviewed in this issue, Nghiem and Kornegay (2019) present the promises and challenges of gene therapy strategies in humans and dogs with DMD. The dog may provide a better animal model than mice for human DMD, as DMD-affected dogs show more similarities to the human clinical and immunological disease progression (Nghiem and Kornegay 2019).

\section{New discoveries for canine inherited diseases}

This special issue of Human Genetics contains several new discoveries related to the molecular basis for genetic diseases in dogs. Human genes for which there is a known variant linked to disease make obvious candidates for similar diseases in dogs. This candidate gene approach can significantly reduce the time from study concept to bona fide mutation in an orthologous canine gene. In addition, many canine researchers are now using whole genome sequencing to identify causal variants in dogs and novel canine genes can be used as candidates and models for human diseases.

In this issue of Human Genetics, Meurs et al. (2019) present the findings of a novel missense variant in the TTN gene that contributes to dilated cardiomyopathy in Doberman pinscher dogs. Variants in TTN are known to contribute to hypertrophic and dilated cardiomyopathies in humans. This discovery in Doberman pinschers helps close the gap in the observed heterogeneity contributing to dilated cardiomyopathy in this breed.

Hytönen et al. (2019) studied Parson Russell terriers and Akita dogs with amelogenesis imperfecta (AI) and identified a variation in the ENAM gene that was found in about $9 \%$ of
Parson Russell terriers studied and a variation in ACP4 in about $22 \%$ of Akita dogs studied that segregated with disease in an autosomal recessive manner. Unlike rodents that have continuously erupting incisors, the physiology of tooth development in dogs more closely reflects that in humans, providing an excellent model organism for studying AI. Both ENAM and ACP4 are genes known to be involved in the pathophysiology of amelogenesis in humans.

In a second paper in this issue, Hytönen and Lohi (2019), using genome-wide homozygosity mapping and whole genome sequencing, identified a variant in the $S G K 3$ gene that segregated with hairlessness in the Scottish Deerhound. Although $S G K 3$ has not been linked to hair abnormalities in humans, it remains a strong candidate for congenital hypotrichosis and early alopecia in humans based on this spontaneous canine model.

Startle disease, or hyperekplexia, is characterized by hypertonia and apnea that are triggered by noise and/or touch. Several causative variants are known in humans and in this issue of Human Genetics, Murphy et al. (2019) identified a novel mutation in the SLC6A5 gene in a family of Spanish Greyhound dogs. Using a candidate gene approach based on previously identified causal variants in humans and mice, this study illustrates how the genetic basis of simple Mendelian disorders in inbred dogs sometimes can be easily elucidated (Murphy et al. 2019).

It is the role and responsibility of the clinical laboratory to evaluate and translate these discoveries into clinical tests to make them available to breeders, veterinarians and dog owners to improve breeding programs and the overall health of dogs. The laboratory can also serve as a resource for researchers, as understanding the clinical validity and utility of a mutation can be challenging and may go beyond what most research laboratories can accomplish. Having a diagnostic test in more than one clinical laboratory allows the testing of a large number of dogs, which can provide better genotype-phenotype correlations among the affected breeds.

In collaboration with a clinical laboratory, Pindar and Ramirez (2019) evaluated serum ALT levels and hepatic copper concentrations and histopathology from ten offspring in which both parents had a least one copy of an $A T P 7 B$ mutation previously shown to be a risk factor for copper toxicosis in Labrador retrievers. None had increased serum ALT activity but all offspring who were homozygous for the $A T P 7 B$ mutation had elevated hepatic copper concentrations. This preliminary study demonstrates that dogs homozygous for the $A T P 7 B$ variant can have abnormally elevated hepatic copper levels despite having normal serum ALT activity. 


\section{Standards and guidelines for clinical canine genetic testing laboratories}

Much like in human clinical genetics, genetic testing in dogs can be used to inform, providing a means for avoiding many inherited diseases. However, unlike human testing, dog breeders can also use genetic testing to understand the genotypes for many traits and coat colors to increase their likelihood of producing dogs with certain qualities desired by puppy buyers. It is for these reasons that the laboratory is not only providing important health information for the dog itself, but also providing useful results that support breeding programs helping to ensure healthier successive generations. As these laboratories are direct-to-consumer, the canine genetic testing laboratory serves as a resource for breeders and veterinarians and must have transparency and clear communication of testing information on their website, reports and provide genetic counseling as needed.

Currently, there is no regulatory oversight for canine clinical genetic testing and no consistency regarding quality assurance within the testing community. The purpose of standards and guidelines is to provide uniformity and guidance towards quality improvement across laboratories in the industry. The first way to achieve this is for the industry as a whole to commit to improving processes that can provide accurate results to the consumer. The second way is for the individual laboratories to implement protocols that help ensure high-quality testing and for providing critical resources to the customer. In this issue of Human Genetics, standards and guidelines have been proposed to assist in guiding the industry as a whole towards common, achievable standards (Shaffer et al. 2018) with a checklist that can be used for self-assessment and continual improvement within individual laboratories (Shaffer et al. 2019). Both the standards and guidelines and the checklist form a baseline for testing standards, with the goal of bringing together the clinical laboratories to continue to discuss and improve these documents to provide consistency and high accuracy across the entire industry. These publications (Shaffer et al. 2018, 2019) are open access, available to all laboratories, and should assist in providing a structure by which the laboratories can begin to develop or enhance their quality assurance programs under which new tests are developed, validated and launched.

In summary, this special issue of Human Genetics dedicated to canine genetics demonstrates an active research community investigating diseases and traits in dogs. Many of these discoveries can serve as animal models for human disease and can be developed into actionable clinical tests that can be used by breeders and veterinarians to improve the health of dogs. Implementation of the new standards and guidelines for clinical testing laboratories will pave the way to uniformity within the testing community and increased accuracy across laboratories.

\section{Compliance with ethical standards}

Conflict of interest LGS is the owner of Genetic Veterinary Sciences, DBA Paw Print Genetics, which provides canine genetic testing for inherited disorders on a fee-for-service basis to breeders, owners and veterinarians.

\section{References}

Bunel M, Chaudieu G, Hamel C, Lagoutte L, Manes G, Botherel N, Brabet P, Pilorge P, André C, Quignon P (2019) Natural models for retinitis pigmentosa: progressive retinal atrophy in dogs breeds. Hum Genet. https://doi.org/10.1007/s00439-019-01999-6

Corréard S, Jocelyn J, Lagoutte L, Botherel N, Thibaud J-L, Hédan $\mathrm{B}$, Richard L, Lia A-S, Delague V, Mège C, Mathis S, Guaguère E, Paradis M, Vallat J-M, Quignon P, André C (2019) Canine neuopathies: powerful spontaneous models for human hereditary sensory neuropathies. Hum Genet. https://doi.org/10.1007/s0043 9-019-02003-x

Hytönen MK, Lohi H (2019) A frameshift insertion in SGK3 leads to recessive hairlessness in Scottish Deerhounds: a candidate gene for human alopecia conditions. Hum Genet. https://doi. org/10.1007/s00439-019-02005-9

Hytönen MK, Arumilli M, Sarkiala E, Nieminen P, Lohi H (2019) Canine models of human amelogenesis imperfecta: identification of novel recessive ENAM and ACP4 variants. Hum Genet. https ://doi.org/10.1007/s00439-019-01997-8

Lindblad-Toh K, Wade CM, Mikkelsen TS, Karlsson EK, Jaffe DB, Kamal M, Clamp M et al (2005) Genome sequence, comparative analysis and haplotype structure of the domestic dog. Nature 438:803-819

Mealey KL, Martinez SE, Villarino NF, Court MH (2019) Personalized medicine: going to the dogs? Hum Genet. https://doi.org/10.1007/ s00439-019-02020-w

Meurs KM, Friedenberg SG, Kolb J, Saripalli C, Tonino P, Woodruff K, Olby NJ, Keene BW, Adin DB, Yost OL, DeFrancesco TC, Lahmers S, Tou S, Shelton GD, Granzier H (2019) A missense variant in the titin gene in Doberman pinscher dogs with familial dilated cardiomyopathy and sudden cardiac death. Hum Genet. https://doi.org/10.1007/s00439-019-01973-2

Murphy SC, Recio A, de la Fuente C, Guo LT, Shelton GD, Clark LA (2019) A glycine transporter SLC6A5 frameshift mutation causes startle disease in Spanish greyhounds. Hum Genet. https://doi. org/10.1007/s00439-019-01986-x

Nghiem PP, Kornegay JN (2019) Gene therapies in canine models for Duchenne muscular dystrophy. Hum Genet. https://doi. org/10.1007/s00439-019-01976-z

Online Mendelian Inheritance in Animals (2019) https://omia.org/ results/?search_type $=$ advanced\&gb_species_id $=9615 \&$ chara cterised=yes. Accessed 20 April 2019

Pindar S, Ramirez J (2019) Predicting copper toxicosis: relationship between the ATP7A and ATP7B gene mutations and hepatic copper quantification in dogs. Hum Genet. https://doi.org/10.1007/ s00439-019-02010-y

Shaffer LG, Sundin K, Geretschlaeger A, Segert J, Swinburne JE, Royal R, Loechel R, Ramirez CJ, Ballif BC (2018) Standards and guidelines for canine clinical genetic testing laboratories. Hum Genet. https://doi.org/10.1007/s00439-018-1954-4 
Shaffer LG, Geretschlaeger A, Ramirez CJ, Ballif BC, Carl C (2019) Quality assurance checklist and additional considerations for canine clinical genetic testing laboratories: a follow-up to the published standards and guidelines. Hum Genet. https://doi. org/10.1007/s00439-019-02013-9
Publisher's Note Springer Nature remains neutral with regard to jurisdictional claims in published maps and institutional affiliations. 